\title{
Oscillation Criteria of First Order Neutral Delay Differential Equations with Variable Coefficients
}

\author{
Fatima N. Ahmed, Rokiah Rozita Ahmad, \\ Ummul Khair Salma Din, and Mohd Salmi Md Noorani
}

School of Mathematical Sciences, Faculty of Science and Technology, Universiti Kebangsaan, 43600 UKM Bangi, Selangor, Malaysia

Correspondence should be addressed to Fatima N. Ahmed; zahra80zahra@yahoo.com

Received 2 May 2013; Accepted 2 September 2013

Academic Editor: Rodrigo Lopez Pouso

Copyright (C) 2013 Fatima N. Ahmed et al. This is an open access article distributed under the Creative Commons Attribution License, which permits unrestricted use, distribution, and reproduction in any medium, provided the original work is properly cited.

Some new oscillation criteria are given for first order neutral delay differential equations with variable coefficients. Our results generalize and extend some of the well-known results in the literature. Some examples are considered to illustrate the main results.

\section{Introduction}

In recent years, oscillation of neutral delay differential equations (or NDDEs for short) has received great attention and has been studied extensively. It is a relatively new field with interesting applications from the real world. In fact, NDDEs appear in modeling of the problems as transformation of information, population dynamics, the networks containing lossless transmission lines, and in the theory of automatic control (see, e.g., [1-4] and references cited therein).

Consider the first order NDDE of the form

$$
[r(t)(x(t)+p(t) x(t-\tau))]^{\prime}+q(t) x(t-\sigma)=0, \quad t \geq t_{0},
$$

where

$$
p \in C\left[\left[t_{0}, \infty\right), \mathbb{R}\right], \quad r, q \in C\left[\left[t_{0}, \infty\right), \mathbb{R}^{+}\right], \quad \tau, \sigma \in \mathbb{R}^{+} .
$$

Let $m=\max \{\tau, \sigma\}$. By a solution of (1), we mean a function $x \in C\left[\left[t_{1}-m, \infty\right), \mathbb{R}\right]$ for some $t_{1} \geq t_{0}$ such that $x(t)+p(t) x(t-\tau)$ is continuously differentiable, and (1) is satisfied identically for $t_{1} \geq t_{0}$. Such a solution of (1) is said to be oscillatory if it has arbitrarily large zeros and nonoscillatory if it is eventually positive or eventually negative. The NDDE (1) is called oscillatory if all its solutions are oscillatory; otherwise, it is called nonoscillatory.
Recently, some investigations such as [5-7] have appeared which are concerned with the oscillation as well as the nonoscillation behaviour of NDDE (1). In fact, Zahariev and Bainnov [8] is the first work dealing with oscillation of neutral equations. A systematic development of oscillation theory of NDDEs was initiated by Ladas and Sficas [9]. For the oscillation of (1) when $r(t)=1$ and $p(t)$ and $q(t)$ are constants, we refer the readers to the articles by Ladas and Schults [10], Sficas and Stavroulakis [11], Grammatikopoulos et al. [12], Zhang [13], and Gopalsamy and Zhang [14]. For the oscillation of (1) when $r(t)=1$ and $p(t)$ is equal to a constant, we refer the readers to the papers by Grammatikopoulos et al. [15], Zhang [13], Gopalsamy and Zhang [14], and Saker and Elabbasy [16] and the references cited therein. Grammatikopoulos et al. [6], Ladas and Schults [10], Chuanxi and Ladas [17, 18], Kubiaczyk and Saker [19], and Karpuz and Ocalan [20] considered the NDDE (1) when $r(t)=1$ and established some new oscillation results sorted by the value of function $p(t)$. For further oscillation results on the oscillatory behaviour of solutions of (1), we refer the readers to the monographs by Györi and Ladas [21] and Erbe et al. [22] as well as the papers of Yu et al. [23], Choi and Koo [24], Ocalan [25], and Candan and Dahiya [26].

The purpose of this work is to find some sufficient conditions for the oscillation of all solutions of the first order NDDE (1). 
Remark 1. (i) When we write a functional inequality we assume that it holds for all sufficiently large $t$.

(ii) Without loss of generality, we will deal only with the positive solutions of (1).

In the proof of our main results, we need the following well-known lemmas which can be found in Chuanxi and Ladas [17], Györi and Ladas [21], and Kulenović et al. [27].

Lemma 2. Assume that $\rho$ is a positive constant. Let $h \in$ $C\left[\left[t_{0}, \infty\right), \mathbb{R}^{+}\right]$, and suppose that

$$
\lim _{t \rightarrow \infty} \inf \int_{t-\rho}^{t} h(s) d s>\frac{1}{e} .
$$

Then

(i) the delay differential inequality

$$
x^{\prime}(t)+h(t) x(t-\rho) \leq 0, \quad t \geq t_{0},
$$

has no eventually positive solution;

(ii) the delay differential inequality

$$
x^{\prime}(t)+h(t) x(t-\rho) \geq 0, \quad t \geq t_{0},
$$

has no eventually negative solution;

(iii) the advanced differential inequality

$$
x^{\prime}(t)-h(t) x(t+\rho) \leq 0, \quad t \geq t_{0},
$$

has no eventually negative solution;

(iv) the advanced differential inequality

$$
x^{\prime}(t)-h(t) x(t+\rho) \geq 0, \quad t \geq t_{0},
$$

has no eventually positive solution.

Lemma 3. Consider the NDDE

$$
(x(t)+p(t) x(t-\tau))^{\prime}+q(t) x(t-\sigma)=0, \quad t \geq t_{0},
$$

where $p, q, \tau$, and $\sigma$ are as in (2). Assume that

$$
\int_{t_{0}}^{\infty} q(s) d s=\infty
$$

Let $x(t)$ be an eventually positive solution of equation and set

$$
z(t)=x(t)+p(t) x(t-\tau)
$$

Then the following statements are true:

(i) $z(t)$ is an eventually decreasing function; (ii) if $p(t) \leq-1$ then $z(t)<0$;

(iii) if $-1 \leq p(t) \leq 0$ then $z(t)>0$ and $\lim _{t \rightarrow \infty} z(t)=0$.

Lemma 4. Assume that (9) holds and let $x(t)$ be an eventually positive solution of NDDE

$$
[(x(t)+p x(t-\tau))]^{\prime}+q(t) x(t-\sigma)=0, \quad t \geq t_{0},
$$

where $p \neq 1, q \in C\left[\left[t_{0}, \infty\right), \mathbb{R}^{+}\right]$, and $\tau, \sigma \in \mathbb{R}^{+}$.

Set

$$
z(t)=x(t)+p x(t-\tau)
$$

Then

(a) $z(t)$ is a decreasing function and either

$$
\lim _{t \rightarrow \infty} z(t)=-\infty
$$

or

$$
\lim _{t \rightarrow \infty} z(t)=0
$$

(b) The following statements are equivalent:

(i) (13) holds;

(ii) $p<-1$;

(iii) $\lim _{t \rightarrow \infty} x(t)=\infty$;

(iv) $w(t)>0, w^{\prime}(t)>0$.

(c) The following statements are equivalent:

(i) (14) holds;

(ii) $p>-1$;

(iii) $\lim _{t \rightarrow \infty} x(t)=0$;

(iv) $w(t)>0, w^{\prime}(t)<0$.

\section{Main Results}

In this section we give some new sufficient conditions for all solutions of NDDE (1) to be oscillatory.

Theorem 5. Assume that (2) and (9) hold, $p(t) \leq-1, \tau>\sigma$, and

$$
\lim _{t \rightarrow \infty} \inf \int_{t+\sigma}^{t+\tau}\left[\frac{q(s-\tau)}{-r(s-\sigma) p(s-\sigma)}\right] d s>\frac{1}{e}
$$

Then every solution of NDDE (1) is oscillatory.

Proof. Assume, for the sake of a contradiction, that (1) has an eventually positive solution $x(t)>0$ for all $t \geq t_{0}>0$. Set

$$
z(t)=x(t)+p(t) x(t-\tau)
$$

Then by Lemma 3 we have

$$
z(t)<0
$$


Observe that

$$
z(t)>p(t) x(t-\tau)
$$

From which we find eventually

$$
\frac{1}{p(t+\tau-\sigma)} q(t) z(t+\tau-\sigma)<q(t) x(t-\sigma)=-(r(t) z(t))^{\prime},
$$

and hence

$$
z^{\prime}(t)+\frac{r^{\prime}(t)}{r(t)} z(t)+\frac{q(t)}{r(t) p(t+\tau-\sigma)} z(t+\tau-\sigma)<0 .
$$

Set

$$
z(t)=e^{-\int_{t_{0}}^{t}\left(r^{\prime}(s) / r(s)\right) d s} y(t) .
$$

This implies that $y(t)<0$.

Substituting in (20) yields for all $t \geq t_{0}$

$$
y^{\prime}(t)+\frac{q(t)}{r(t+\tau-\sigma) p(t+\tau-\sigma)} y(t+\tau-\sigma)<0,
$$

or

$$
y^{\prime}(t)-\left[\frac{q(t)}{-r(t+\tau-\sigma) p(t+\tau-\sigma)}\right] y(t+(\tau-\sigma))<0 .
$$

In view of (15) and Lemma 2(iii), it is impossible for (23) to have an eventually negative solution. This contradicts the fact that $y(t)<0$ and the proof is complete.

Example 6. Consider NDDE

$$
\left[\frac{e^{t+1}}{t+1}\left(x(t)-\frac{t+1}{t} x(t-2)\right)\right]^{\prime}+e^{t+2} x(t-1)=0, \quad t>0 .
$$

Here we have

$$
\begin{gathered}
p(t)=-\frac{t+1}{t} \leq-1, \quad q(t)=e^{t+2}, \\
r(t)=\frac{e^{t+1}}{t+1}, \quad \tau=2, \quad \sigma=1 .
\end{gathered}
$$

Then all the hypotheses of Theorem 5 are satisfied where

$$
\begin{aligned}
& \lim _{t \rightarrow \infty} \inf \int_{t+\sigma}^{t+\tau} \frac{q(s-\tau)}{-r(s-\sigma) p(s-\sigma)} d s \\
& \quad=\lim _{t \rightarrow \infty} \inf \int_{t+1}^{t+2}(s-1) d s=\lim _{t \rightarrow \infty} \inf \left(t+\frac{9}{2}\right)=\infty>\frac{1}{e} .
\end{aligned}
$$

Hence every solution of (24) is oscillatory.

Remark 7. Theorem 5 is an extent of [17, Theorem 2], [15, Theorem 7], and [21, Theorem 6.4.3].
Theorem 8. Assume that (2) and (9) hold, $-1 \leq p(t) \leq 0$, and

$$
\lim _{t \rightarrow \infty} \inf \int_{t-\sigma}^{t} \frac{q(s)}{r(s-\sigma)} d s>\frac{1}{e}
$$

Then every solution of NDDE (1) oscillates.

Proof. Assume, for the sake of contradiction, that (1) has an eventually positive solution $x(t)>0$ for all $t \geq t_{0}>0$. Set

$$
z(t)=x(t)+p(t) x(t-\tau) .
$$

Then by Lemma 3, it follows that

$$
z(t)>0 \text {. }
$$

As $x(t)>z(t)$, it follows from (1) that

$$
(r(t) z(t))^{\prime}+q(t) z(t-\sigma) \leq 0 .
$$

Dividing the last inequality by $r(t)>0$, we obtain

$$
z^{\prime}(t)+\frac{r^{\prime}(t)}{r(t)} z(t)+\frac{q(t)}{r(t)} z(t-\sigma) \leq 0 .
$$

Let

$$
z(t)=e^{-\int_{t_{0}}^{t}\left(r^{\prime}(s) / r(s)\right) d s} y(t) .
$$

This implies that $y(t)>0$.

Substituting in (31) yields for all $t \geq t_{0}$

$$
y^{\prime}(t)+\frac{q(t)}{r(t-\sigma)} y(t-\sigma) \leq 0, \quad t \geq t_{0} .
$$

In view of Lemma 2(i) and (27), it is impossible for (33) to have an eventually positive solution. This contradicts the fact that $y(t)>0$ and the proof is complete.

Example 9. Consider the NDDE

$$
\begin{array}{r}
{\left[\frac{1}{t}\left(x(t)-\frac{t}{t+1} x(t-\tau)\right)\right]^{\prime}+\frac{1}{t-(5 \pi / 2)} x\left(t-\frac{5 \pi}{2}\right)=0,} \\
t>\frac{5 \pi}{2} .
\end{array}
$$

Note that all the hypotheses of Theorem 8 are satisfied:

$$
\lim _{t \rightarrow \infty} \inf \int_{t-\sigma}^{t} \frac{q(s)}{r(s-\sigma)} d s=\lim _{t \rightarrow \infty} \inf \int_{t-(5 \pi / 2)}^{t} d s=\frac{5 \pi}{2}>\frac{1}{e} .
$$

Therefore every solution of (34) is oscillatory.

Remark 10. Theorem 8 is an extent of [17, Theorem 3] and [21, Theorem 6.4.2].

Theorem 11. Assume that (2) holds with $p(t) \equiv p \neq \pm 1, r(t) \equiv$ $r>0, q(t)$ being $\tau$ periodic, and

$$
\frac{1}{r(1+p)} \lim _{t \rightarrow \infty} \inf \int_{t-\sigma}^{t-\tau} q(s) d s>\frac{1}{e} .
$$


Then every solution of NDDE

$$
[r(x(t)+p x(t-\tau))]^{\prime}+q(t) x(t-\sigma)=0, \quad t \geq t_{0},
$$

is oscillatory.

Proof. Assume, for the sake of contradiction, that (37) has an eventually positive solution $x(t)>0$ for all $t \geq t_{0}>0$. Set

$$
\begin{aligned}
& z(t)=x(t)+p x(t-\tau), \\
& w(t)=z(t)+p z(t-\tau) .
\end{aligned}
$$

It is easily seen, by direct substituting, that $z(t)$ and $w(t)$ are also solutions of (37). That is,

$$
\begin{gathered}
r z^{\prime}(t)+p r z^{\prime}(t-\tau)+q(t) z(t-\sigma)=0, \\
r w^{\prime}(t)+p r w^{\prime}(t-\tau)+q(t) w(t-\sigma)=0 .
\end{gathered}
$$

By Lemma $4, z(t)$ is decreasing and either (13) or (14) holds. In either case we claim that

$$
w^{\prime}(t-\tau) \geq w^{\prime}(t) .
$$

Indeed,

$$
\begin{aligned}
w^{\prime}(t) & =-\frac{1}{r} q(t) z(t-\sigma) \leq-\frac{1}{r} q(t) z(t-\sigma-\tau) \\
& =-\frac{1}{r} q(t-\tau) z(t-\sigma-\tau)=w^{\prime}(t-\tau) .
\end{aligned}
$$

Furthermore, we have by Lemma 4 that as long as $p \neq \pm 1$,

$$
w(t)>0
$$

Using (41) in (40) implies

$$
r(1+p) w^{\prime}(t-\tau)+q(t) w(t-\sigma) \leq 0
$$

or

$$
w^{\prime}(t-\tau)+\frac{1}{r(1+p)} q(t) w(t-\sigma) \leq 0 .
$$

Since $q(t)$ is periodic of period $\tau$, we find

$$
w^{\prime}(t)+\frac{1}{r(1+p)} q(t) w(t-(\sigma-\tau)) \leq 0, \quad \text { if } 1+p>0
$$

or

$$
\begin{array}{r}
w^{\prime}(t)-\left[\frac{1}{-r(1+p)}\right] q(t) w(t+(\tau-\sigma)) \geq 0 \\
\text { if } 1+p<0 .
\end{array}
$$

In view of Lemma 2((i) and (iv)) and (36), it is impossible for (46) and (47) to have eventually positive solutions. This contradicts the fact that $w(t)>0$ and the proof is complete.

Remark 12. Theorem 11 extends [15, Theorems 8 and 10]. See also [21, Theorem 6.4.4].

\section{Acknowledgment}

This research has been completed with the support of these grants: ukm-DLP-2011-049, DIP-2012-31, and FRGS/1/2012/ SG04/ukm/01/1.

\section{References}

[1] H. A. Agwo, "On the oscillation of delay differential equations with real coefficients," International Journal of Mathematics and Mathematical Sciences, vol. 22, no. 3, pp. 573-578, 1999.

[2] R. D. Driver, "A mixed neutral system," Nonlinear Analysis: Theory, Methods \& Applications, vol. 8, no. 2, pp. 155-158, 1984.

[3] J. K. Hale, Theory of Functional Differential Equations, vol. 3 of Applied Mathematical Sciences, Springer, New York, NY, USA, 1977.

[4] N. Parhi and R. N. Rath, "On oscillation and asymptotic behaviour of solutions of forced first order neutral differential equations," Indian Academy of Sciences, vol. 111, no. 3, pp. 337350, 2001.

[5] R. P. Agarwal, M. Bohner, and W. T. Li, Nonoscillation and Oscillation: Theory for Functional Differential Equations, vol. 267 of Monographs and Textbooks in Pure and Applied Mathematics, Marcel Dekker, New York, NY, USA, 2004.

[6] M. K. Grammatikopoulos, G. Ladas, and Y. G. Sficas, "Oscillation and asymptotic behavior of neutral equations with variable coefficients," Radovi Matematicki, vol. 2, no. 2, pp. 279-303, 1986.

[7] S. Tanaka, "Oscillation of solutions of first-order neutral differential equations," Hiroshima Mathematical Journal, vol. 32, no. 1, pp. 79-85, 2002.

[8] A. I. Zahariev and D. D. Bainnov, "Oscillating properties of the solutions of a class of neutral type functional-differential equations," Bulletin of the Australian Mathematical Society, vol. 22 , no. 3, pp. 365-372, 1980 .

[9] G. Ladas and Y. G. Sficas, "Oscillations of neutral delay differential equations," Canadian Mathematical Bulletin, vol. 29, no. 4, pp. 438-445, 1986.

[10] G. Ladas and S. W. Schults, "On oscillations of neutral equations with mixed arguments," Hiroshima Mathematical Journal, vol. 19, no. 2, pp. 409-429, 1989.

[11] Y. G. Sficas and I. P. Stavroulakis, "Necessary and sufficient conditions for oscillations of neutral differential equations," Journal of Mathematical Analysis and Applications, vol. 123, no. 2, pp. 494-507, 1987.

[12] M. K. Grammatikopoulos, E. A. Grove, and G. Ladas, "Oscillation and asymptotic behavior of neutral differential equations with deviating arguments," Applicable Analysis, vol. 22, no. 1, pp. $1-19,1986$.

[13] B. G. Zhang, "Oscillation of first order neutral functionaldifferential equations," Journal of Mathematical Analysis and Applications, vol. 139, no. 2, pp. 311-318, 1989.

[14] K. Gopalsamy and B. G. Zhang, "Oscillation and nonoscillation in first order neutral differential equations," Journal of Mathematical Analysis and Applications, vol. 151, no. 1, pp. 42-57, 1990.

[15] M. K. Grammatikopoulos, E. A. Grove, and G. Ladas, "Oscillations of first-order neutral delay differential equations," Journal of Mathematical Analysis and Applications, vol. 120, no. 2, pp. 510-520, 1986.

[16] S. H. Saker and E. M. Elabbasy, "Oscillation of first order neutral delay differential equations," Kyungpook Mathematical Journal, vol. 41, no. 2, pp. 311-321, 2001. 
[17] Q. Chuanxi and G. Ladas, "Oscillations of neutral differential equations with variable coefficients," Applicable Analysis, vol. 32, no. 3-4, pp. 215-228, 1989.

[18] Q. Chuanxi and G. Ladas, "Oscillations of first-order neutral equations with variable coefficients," Monatshefte für Mathematik, vol. 109, no. 2, pp. 103-111, 1990.

[19] I. Kubiaczyk and S. H. Saker, "Oscillation of solutions to neutral delay differential equations," Mathematica Slovaca, vol. 52, no. 3, pp. 343-359, 2002.

[20] B. Karpuz and O. Ocalan, "Oscillation criteria for some classes of linear delay differential equations of first-order," Bulletin of the Institute of Mathematics, vol. 3, no. 2, pp. 293-314, 2008.

[21] I. Györi and G. Ladas, Oscillation Theory of Delay Differential Equations, Oxford Mathematical Monographs, Clarendon Press, New York, NY, USA, 1991.

[22] L. H. Erbe, Q. Kong, and B. G. Zhang, Oscillation Theory for Functional-Differential Equations, vol. 190 of Monographs and Textbooks in Pure and Applied Mathematics, Marcel Dekker, New York, NY, USA, 1995.

[23] J. S. Yu, Z. C. Wang, and C. X. Qian, "Oscillation of neutral delay differential equations," Bulletin of the Australian Mathematical Society, vol. 45, no. 2, pp. 195-200, 1992.

[24] S. K. Choi and N. J. Koo, "Oscillation theory for delay and neutral differential equations," Trends in Mathematics, Information Center for Mathematical Sciences, vol. 2, pp. 170-176, 1999.

[25] O. Ocalan, "Existence of positive solutions for a neutral differential equation with positive and negative coefficients," Applied Mathematics Letters, vol. 22, no. 1, pp. 84-90, 2009.

[26] T. Candan and R. S. Dahiya, "Positive solutions of first-order neutral differential equations," Applied Mathematics Letters, vol. 22, no. 8, pp. 1266-1270, 2009.

[27] M. R. S. Kulenović, G. Ladas, and A. Meimaridou, "Necessary and sufficient condition for oscillations of neutral differential equations," Journal of the Australian Mathematical Society B, vol. 28, no. 3, pp. 362-375, 1987. 


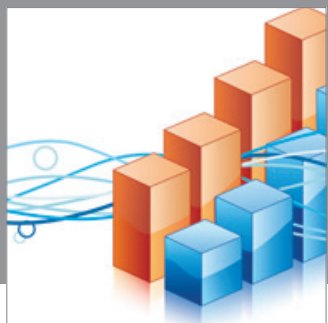

Advances in

Operations Research

mansans

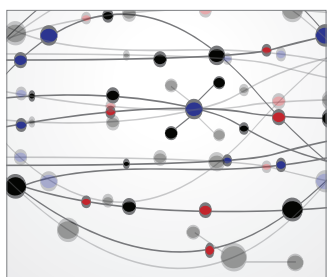

The Scientific World Journal
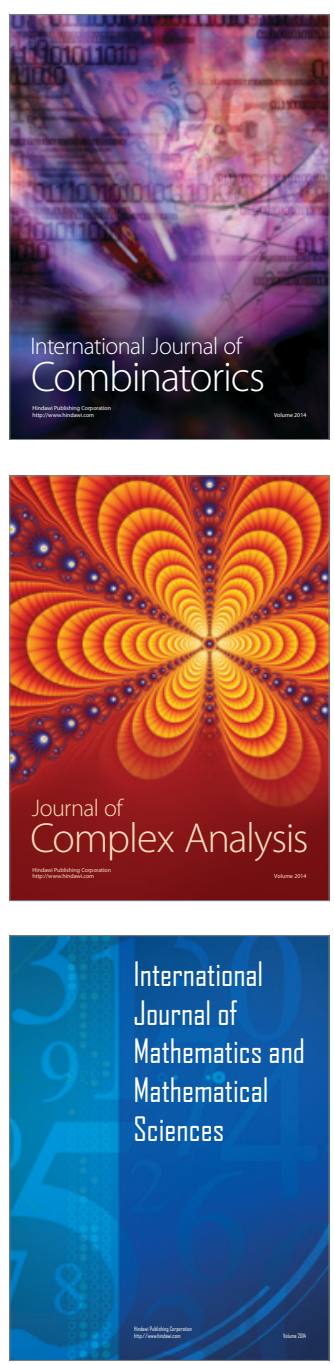
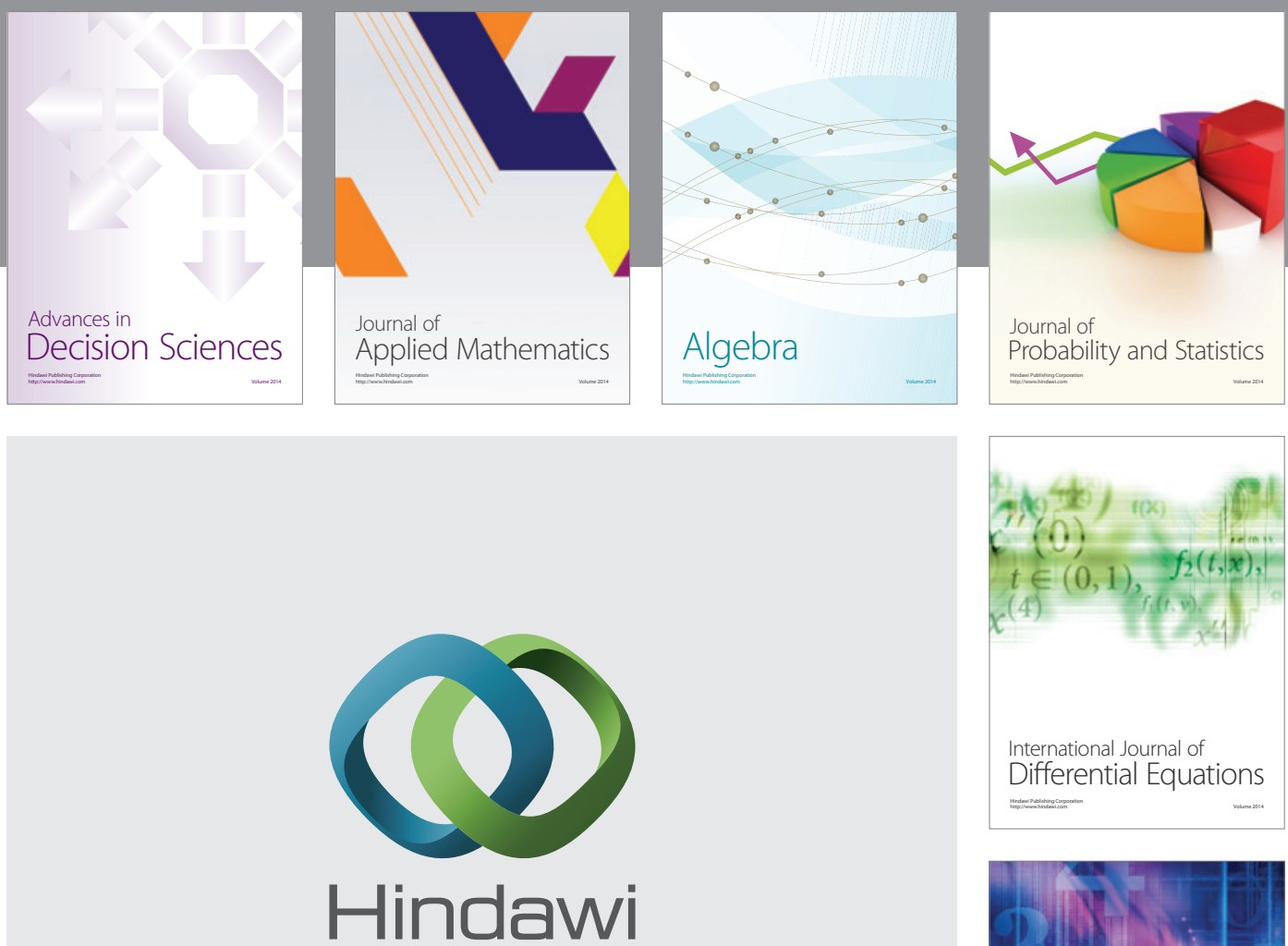

Submit your manuscripts at http://www.hindawi.com
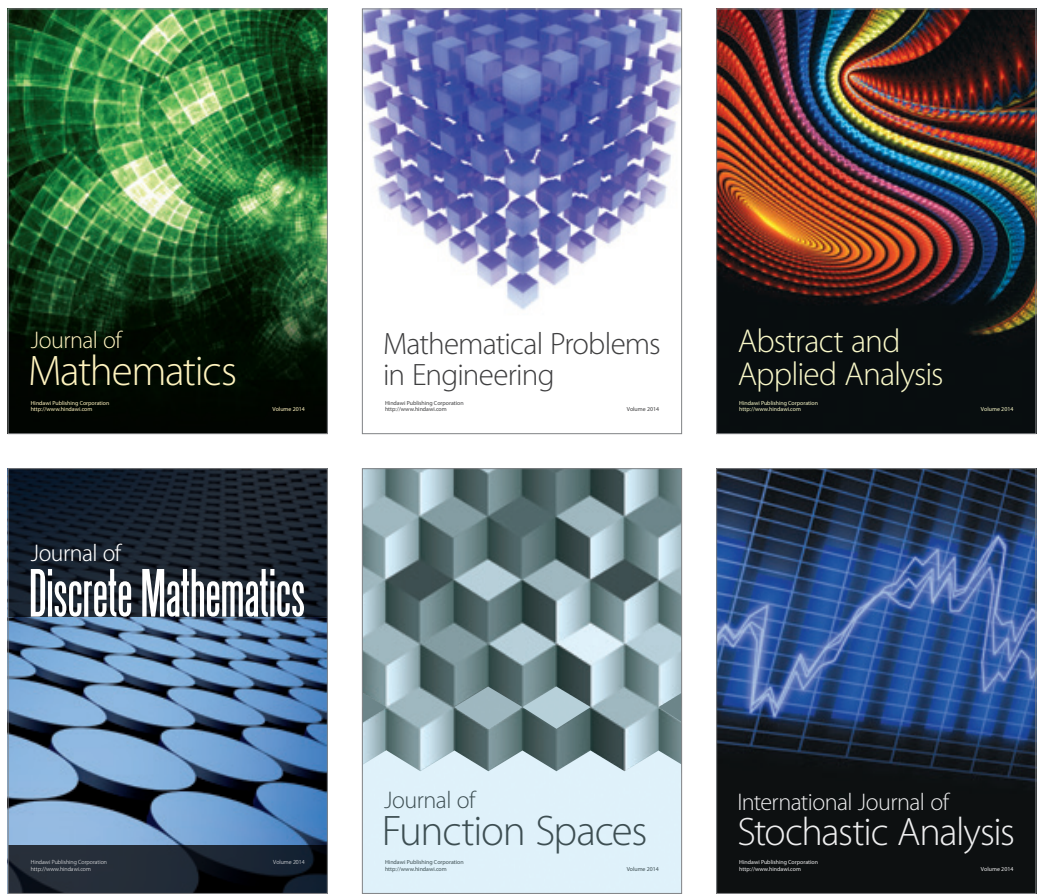

Journal of

Function Spaces

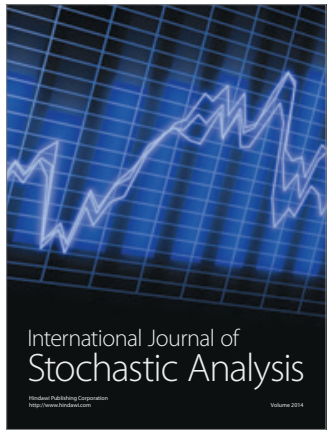

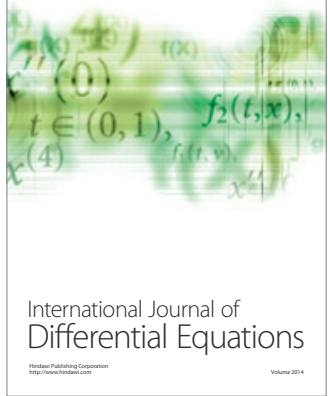
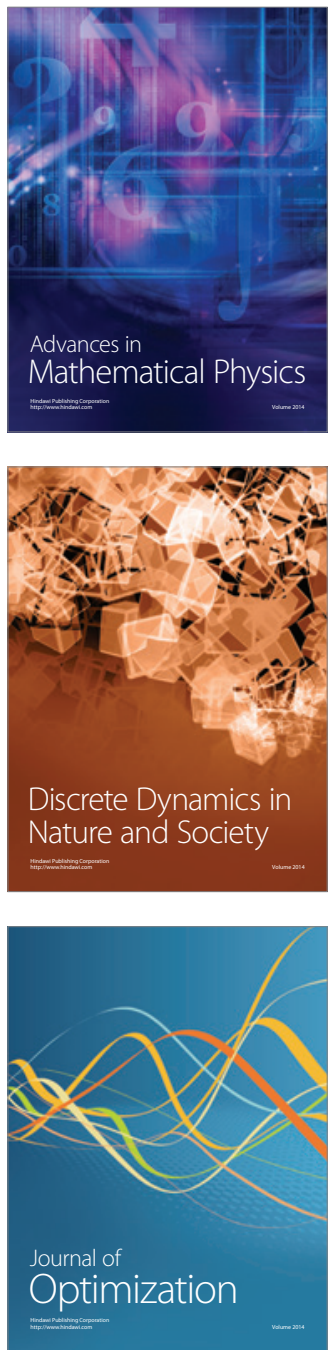